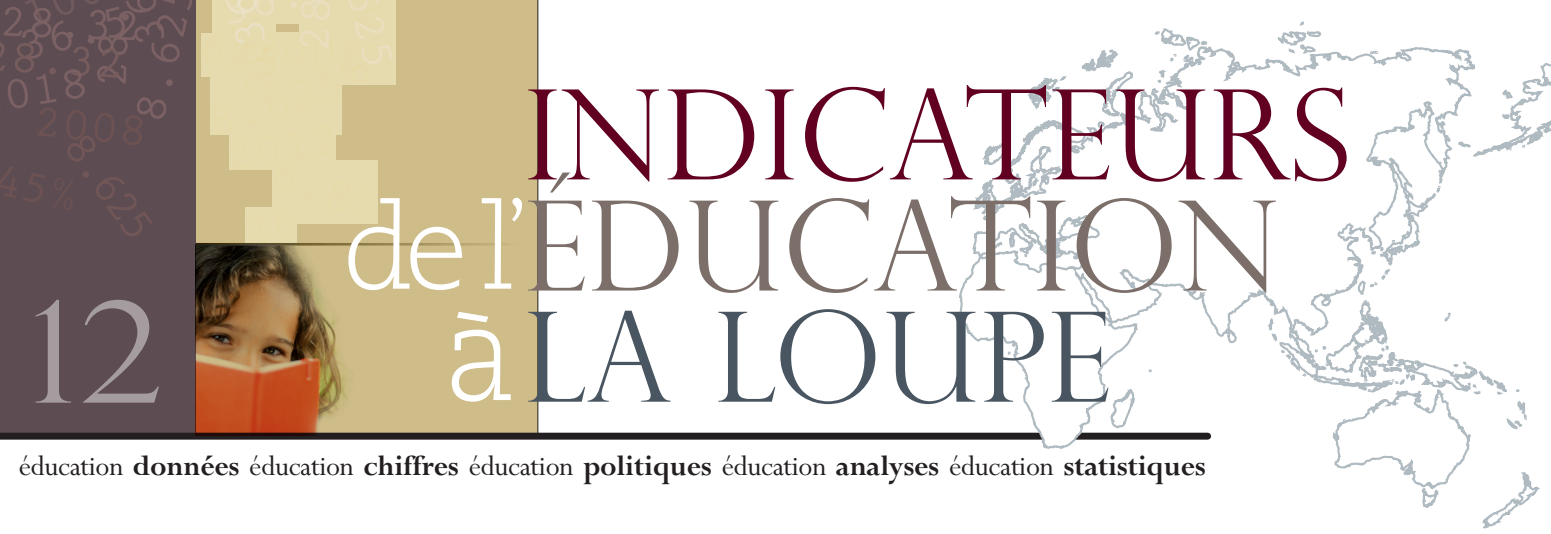

\title{
Quels facteurs influent sur le niveau des dépenses au titre du personnel enseignant?
}

- Le coût salarial des enseignants par élève augmente avec le niveau d'enseignement. En Belgique (Communauté flamande), en Espagne et en France, l'écart de coût salarial annuel entre l'enseignement primaire et le premier cycle de l'enseignement secondaire dépassait 1800 USD selon les chiffres de 2010.

- Entre 2000 et 2010, le coût salarial des enseignants par élève a augmenté dans presque tous les pays, de l'ordre d'un tiers dans l'enseignement primaire et d'un quart dans le premier cycle de l'enseignement secondaire.

- C'est le salaire des enseignants qui est le facteur le plus déterminant de l'évolution du coût salarial ; vient ensuite la taille des classes.

- Des niveaux de dépenses similaires d'un pays à l'autre peuvent masquer des choix politiques contrastés.

Les gouvernements des pays de l'OCDE s'intéressent fortement à la relation entre les moyens mobilisés en faveur de l'éducation et les résultats obtenus par les élèves.

D'une part, les gouvernements cherchent à accroître la scolarisation et à améliorer le niveau de formation de leur population, tandis que d'autre part, dans les conditions actuelles d'austérité budgétaire, il est de la plus haute importance de veiller à l'usage efficace des fonds publics pour parvenir aux résultats escomptés.

Dans l'ensemble des pays de I'OCDE, le coût salarial des enseignants est le poste le plus important du budget affecté à l'éducation, selon la combinaison mathématique de quatre facteurs : le salaire des enseignants, la taille des classes, le nombre d'heures d'enseignement données par les enseignants et le nombre d'heures de d'instruction reçues par les élèves (voir l'encadré B7.1 dans OCDE, 2012).

Ainsi, un niveau donné de dépenses au titre du personnel enseignant peut résulter de différentes combinaisons de ces quatre facteurs. De même, une réforme (ou un changement structurel) de l'un de ces facteurs (tous autres facteurs égaux par ailleurs) a une incidence directe sur le niveau des dépenses: par exemple, l'augmentation du salaire des enseignants suppose d'augmenter le budget, la diminution de la taille des classes entraîne un besoin d'enseignants supplémentaires et donc également une augmentation du budget, ce qui est aussi le cas pour l'augmentation du nombre d'heures d'instruction reçues par les élèves ou la diminution du nombre d'heures d'enseignement données par les enseignants.

Le coût salarial des enseignants par élève augmente avec le niveau d'enseignement, mais on constate de fortes disparités entre les pays de l'OCDE.

Les dépenses au titre de l'éducation évoluent selon un schéma similaire dans les pays de l'OCDE : elles augmentent fortement avec le niveau d'enseignement. En moyenne, dans les pays de l'OCDE dont les données sont disponibles, le coût salarial des enseignants en 2010 s'établissait à 2307 USD par élève de l'enseignement primaire, 2856 USD par élève du premier cycle de l'enseignement secondaire et 3301 USD par élève du deuxième cycle de l'enseignement secondaire (voir le graphique figurant à la page 2). Au Chili et en Hongrie, l'écart entre les différents niveaux d'enseignement est inférieur à 50 USD, mais il est supérieur à 1800 USD en Belgique (Communauté française), en France et au Portugal, et passe la barre des 2000 USD en Belgique (Communauté flamande). 
Coût salarial (en USD) des enseignants par élève, selon le niveau d'enseignement (2010)

\begin{tabular}{lllll}
\hline USD \\
12000 \\
\hline 10000 \\
\hline 8000
\end{tabular}

Les pays sont classés par ordre décroissant du coût salarial des enseignants par élève dans le premier cycle de l'enseignement secondaire. Source : OCDE, Regards sur l'éducation 2012 : Les indicateurs de l'OCDE, indicateur B7 (www.oecd.org/edu/rse2012).

Ce schéma résulte en partie de l'augmentation du salaire des enseignants et du nombre d'heures d'instruction reçues par les élèves aux niveaux d'enseignement plus élevés (voir le tableau ci-dessous). Une autre explication réside dans la tendance générale à une diminution du temps d'enseignement à mesure que le niveau d'enseignement augmente (il faut donc davantage d'enseignants pour donner cours à un nombre donné d'élèves). L'accroissement de la taille des classes aux niveaux supérieurs d'enseignement a tendance à diminuer le coût salarial par élève. Ces différences entre les pays sont également à mettre au compte des écarts de niveau de leur richesse nationale.

\section{Moyenne de I'OCDE pour les quatre facteurs composant le coût salarial des enseignants par élève, selon le niveau d'enseignement (2010)}

\begin{tabular}{l|c|c|c} 
& Primaire & Premier cycle du secondaire & Deuxième cycle du secondaire \\
\hline Salaire des enseignants & 38162 USD & 39873 USD & 41182 USD \\
\hline $\begin{array}{l}\text { Taille des classes } \\
\begin{array}{l}\text { Nombre d'heures d'enseignement } \\
\text { en classe données par un enseignant }\end{array}\end{array}$ & 782 heures & 18 élèves & 20 élèves \\
$\begin{array}{l}\text { Nombre d'heures d'instruction } \\
\text { recues par les élèves }\end{array}$ & 844 heures & 704 heures & 658 heures \\
\hline
\end{tabular}

Entre 2000 et 2010, l'augmentation du coût salarial des enseignants par élève a principalement été influencée par des variations du salaire des enseignants et de la taille des classes.

En moyenne, dans les pays dont les données sont disponibles pour les deux années, le coût salarial des enseignants par élève

a augmenté d'un tiers dans l'enseignement primaire et d'un quart dans le premier cycle de l'enseignement secondaire entre 2000 et 2010, passant respectivement de 1733 USD à 2307 USD, et de 2273 USD à 2856 USD. La France et

I'Italie sont les deux seuls pays où le coût salarial des enseignants par élève a (légèrement) diminué sur cette période.

En France, cette tendance est principalement imputable à une baisse des salaires et en Italie, à une diminution du nombre d'heures d'instruction pour les élèves.

Plus généralement, dans l'enseignement primaire comme dans le premier cycle de l'enseignement secondaire, l'augmentation a été notamment influencée par les variations du salaire des enseignants et de la taille des classes. Entre 2000 et 2010, parmi les pays dont les données sont disponibles, le salaire des enseignants (exprimé en prix constants) a augmenté de $16 \%$ dans l'enseignement primaire et de $14 \%$ dans le premier cycle de l'enseignement secondaire, en moyenne, tandis que la taille des classes a diminué d'environ $14 \%$ dans l'enseignement primaire et de $7 \%$ dans le premier cycle de l'enseignement secondaire, en moyenne également. 
18.4 Entre 2000 et 2010, le salaire des enseignants a augmenté en valeur réelle dans la plupart des pays dont les données sont comparables. Les augmentations les plus importantes - dépassant largement 50 \% - ont été constatées en Estonie, en République tchèque et en Turquie. À l'inverse, dans plus de trois quarts des pays, la 52010 taille des classes a eu tendance à décroître dans l'enseignement primaire sur cette même période, notamment République tchèque et en Turquie) (voir le graphique ci-dessous). Dans certains cas, la diminution significative de la taille des classes (conduisant à une augmentation du coût salarial des enseignants) n'est pas intervenue suite à une mesure d'action publique, mais a plutôt résulté de changements démographiques et d'une diminution des effectifs d'élèves.

Dans l'ensemble, les deux autres facteurs (le temps d'instruction et le temps d'enseignement) n'ont guère, voire pas du tout, évolué entre 2000 et 2010. Cette absence d'évolution peut être attribuée au caractère sensible des réformes dans ces domaines. Toutefois, dans un petit nombre de pays, le temps d'instruction et/ou le temps d'enseignement ont bel et bien évolué de façon significative. Dans l'enseignement primaire, c'est en République tchèque que le temps d'enseignement a le plus augmenté (200 heures de plus) (le salaire des enseignants y a également doublé), et en Islande que le temps d'instruction a le plus augmenté (près de 200 heures de plus) entre 2000 et 2010.

Les réformes liées à ces quatre facteurs ont une incidence sur les dépenses d'éducation et peuvent aussi affecter les résultats d'apprentissage. Cependant, la relation entre les dépenses et les résultats est loin d'être évidente. Sur la période 2000-09, les résultats de l'enquête PISA montrent que dans la plupart des pays de l'OCDE, la performance des jeunes de 15 ans n'a pas évolué de façon significative (voir OCDE, 2010), même si des réformes ont été entreprises dans les domaines du temps d'instruction, du temps d'enseignement, de la taille des classes ou du salaire des enseignants. Qui plus est, les réformes portant sur les pratiques pédagogiques peuvent également influencer les résultats d'apprentissage sans nécessairement provoquer d'augmentation ou de diminution des dépenses.

\section{Évolution du coût salarial des enseignants par élève, du salaire des enseignants et de la taille des classes dans l'enseignement primaire et le premier cycle de l'enseignement secondaire (entre 2000 et 2010, en pourcentage)}

\begin{tabular}{|c|c|c|c|c|c|c|c|c|c|c|c|c|c|c|c|c|c|c|c|c|c|c|c|c|c|c|c|c|c|c|c|c|c|}
\hline $\begin{array}{c}\% \\
210 \\
\end{array}$ & & & & & Évo & luti & b1 & le la & tail & le d & $\begin{array}{l}\text { s en } \\
\text { es } \mathrm{Cl}\end{array}$ & sse & & & & & TII & $\mathrm{Cc}$ & 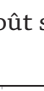 & sala & rial & des & ense & na & ants & ar & c élè & & & & & & $\begin{array}{c}\% \\
210 \\
\end{array}$ \\
\hline 160 & & & & & & & & imá & ire & & & & & & & & $\diamond$ & & & & Pre & mie & $\mathrm{cy}$ & cle & du s & seco & inda & aire & & & & & 160 \\
\hline 110 & & & & & & & & & & & & & & & & & & & & & & & & & & & & & & & & & 110 \\
\hline 60 & & $\diamond$ & $\diamond$ & & & & & & & & & & & & & & & & & & & & & & & & & & & & & & 60 \\
\hline 10 & & & & & (1) & & & & & & & & & & & & & & & & & & & & & & 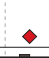 & & & & & & 10 \\
\hline-40 & . & $\square$ & I & T & & & & $\square$ & & & & & & & & & T & $\pi$ & I & & & U & ப & $\square$ & & & & & & & & & -40 \\
\hline & 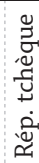 & ن & 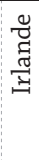 & 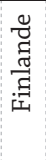 & $\begin{array}{l}\tilde{\sigma} \\
\tilde{\sigma} \\
\omega\end{array}$ & 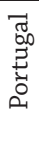 & 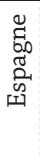 & 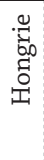 & 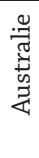 & 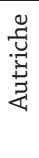 & 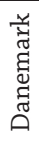 & 党 & 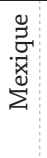 & 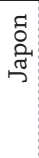 & ॠ & 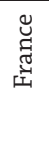 & 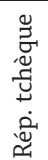 & 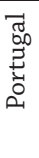 & 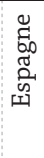 & $\begin{array}{l}\stackrel{\Xi}{\widetilde{E}} \\
\stackrel{\widetilde{\omega}}{\omega}\end{array}$ & 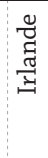 & 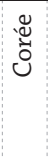 & 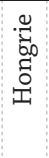 & 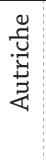 & 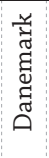 & 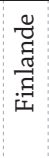 & 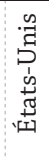 & 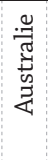 & 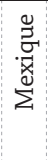 & 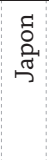 & 章 & 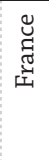 & \\
\hline
\end{tabular}

\section{Des niveaux de dépenses similaires d'un pays à l'autre peuvent masquer} des choix politiques contrastés.

Un niveau de dépenses élevé au titre de l'éducation n'entraîne pas systématiquement de meilleurs résultats des systèmes d'éducation, comme on peut le constater en comparant la performance moyenne des jeunes de 15 ans sur l'échelle PISA 2009 de compétence en compréhension de l'écrit avec les dépenses cumulées par élève entre l'âge de 6 et 15 ans en 2009.

II n'y a là rien d'étonnant car des pays qui dépensent des montants similaires au titre de l'éducation n'ont pas nécessairement les mêmes politiques et pratiques en matière d'éducation. À titre d'exemple, dans le deuxième cycle de l'enseignement secondaire, I'Allemagne et l'Espagne affichent des niveaux similaires de coût salarial des enseignants par élève (soit respectivement 5052 USD et 5100 USD), niveaux tous deux supérieurs à la moyenne de l'OCDE. En Allemagne, ceci est principalement 


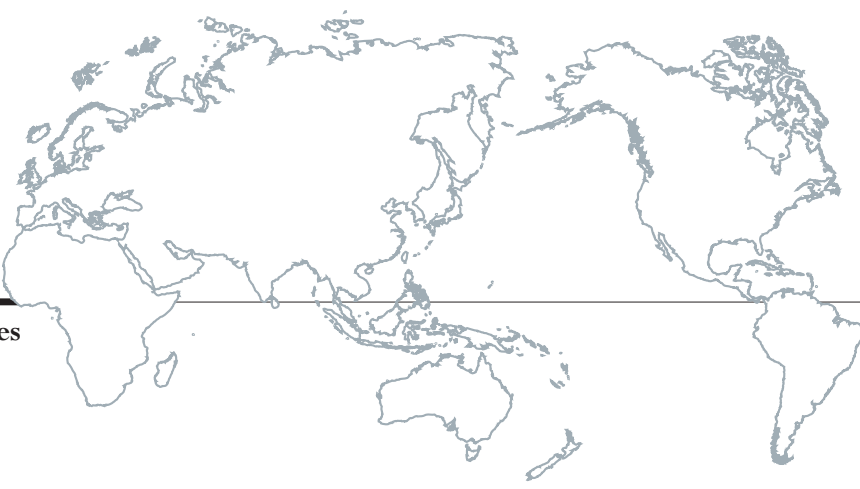

dû au niveau de salaire des enseignants qui est significativement plus élevé que la moyenne de l'OCDE, tandis qu'en Espagne, il s'agit d'une combinaison des facteurs suivants : une taille des classes inférieure à la moyenne, un temps d'instruction supérieur à la moyenne et un salaire des enseignants supérieur à la moyenne.

\section{Contribution (en USD) de divers facteurs au coût salarial des enseignants par élève, dans le deuxième cycle de l'enseignement secondaire (2010)}

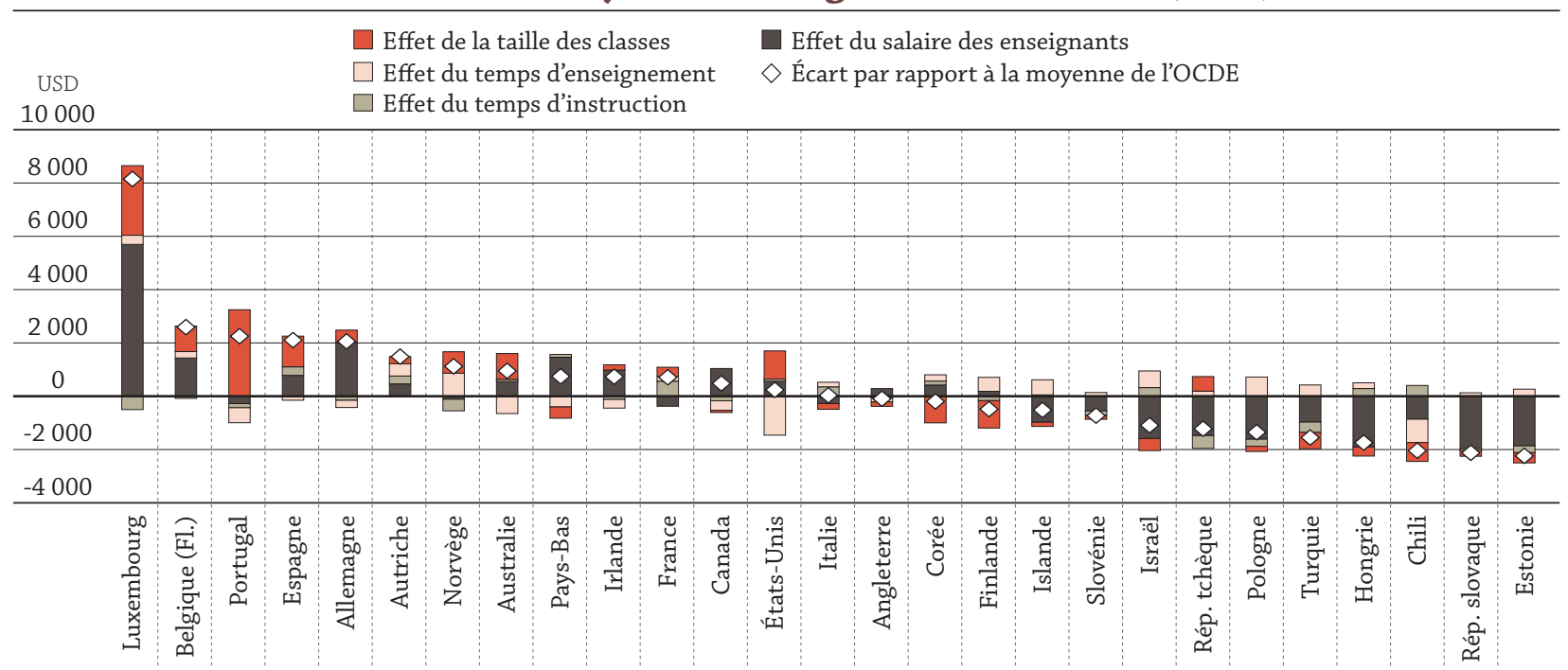

Les pays sont classés par ordre décroissant de lécart entre le coût salarial des enseignants par élève et la moyenne de l'OCDE.

Source : OCDE, Regards sur l'éducation 2012: Les indicateurs de l'OCDE, indicateur B7 (www.oecd.org/edu/rse2012).

Parallèlement à ces contrastes, on relève également d'importantes similitudes dans les choix politiques des pays, même si celles-ci peuvent aboutir à différents niveaux de coût salarial des enseignants par élève. Par exemple, en Australie, aux États-Unis, en Nouvelle-Zélande et au Royaume-Uni, le coût salarial des enseignants par élève du deuxième cycle de l'enseignement secondaire est la conséquence de l'équilibre entre deux effets contraires : un temps d'enseignement supérieur à la moyenne permet de réduire le coût salarial des enseignants par élève par rapport à la moyenne de l'OCDE, tandis qu'une taille des classes relativement faible accroît le coût salarial des enseignants par élève par rapport à la moyenne de I'OCDE. Cependant, le coût salarial des enseignants par élève résultant de cette combinaison est supérieur à la moyenne de I'OCDE en Australie et au Royaume-Uni, mais inférieur à la moyenne de I'OCDE aux États-Unis et en Nouvelle-Zélande, où le temps d'enseignement et la taille des classes sont plus proches de la moyenne de l'OCDE que dans les deux premiers pays.

Pour conclure Dans presque tous les pays, le coût salarial des enseignants du primaire et du premier cycle du secondaire a fortement augmenté entre 2000 et 2010. Cette évolution est principalement due à la hausse du salaire des enseignants et à la diminution de la taille des classes. Des enseignants mieux payés et des classes plus petites ont exercé une pression à la hausse sur les dépenses d'éducation. Les données de l'enquête PISA pour cette période montrent que dans la plupart des pays, ces mesures d'action publique n'ont pas encore entraîné d'amélioration significative des résultats d'apprentissage.

\begin{tabular}{l|l}
$\begin{array}{l}\text { Voir : } \\
\text { www.oecd.org/edu/ } \\
\text { rse2012 }\end{array}$ & $\begin{array}{l}\text { Consulter : } \\
\text { OCDE (2012), Regards sur l'éducation 2012: Les indicateurs }\end{array}$ \\
\hline de l'OCDE, Éditions OCDE. \\
$\begin{array}{l}\text { OCDE (2010), Résultats du PISA 2009: Tendances dans } \\
\text { l'apprentissage : Ĺévolution de la performance des élèves } \\
\text { depuis 2000 (Volume V), PISA, Éditions OCDE. }\end{array}$
\end{tabular}

Pour plus d'informations, Prochain numéro: contacter :

Éric Charbonnier

(Eric.Charbonnier@oecd.org)

et Étienne Albiser

(Etienne.Albiser@oecd.org)
Transition des études

à l'emploi : quelles

perspectives? 